\title{
From evidence to practice: development of web-based Dutch lipid reference values
}

\author{
N. S. Nurmohamed · D. Collard · J. W. Balder · J. A. Kuivenhoven · E. S. G. Stroes · L. F. Reeskamp
}

Accepted: 9 March 2021 / Published online: 12 April 2021

(C) The Author(s) 2021

\begin{abstract}
Introduction In the Netherlands, the total number of yearly measured lipid profiles exceeds 500,000. While lipid values are strongly affected by age and sex, until recently, no up-to-date age- and sex-specific lipid reference values were available. We describe the translation of big-cohort lipid data into accessible reference values, which can be easily incorporated in daily clinical practice.

Methods Lipid values (total cholesterol, LDL cholesterol, HDL cholesterol and triglycerides) from all healthy adults and children in the LifeLines cohort were used to generate age- and sex-specific percentiles. A combination of RStudio, Cascading Style Sheets and HyperText Markup Language was used to interactively display the percentiles in a responsive web layout.

Results After exclusion of subjects reporting cardiovascular disease or lipid-lowering therapy at baseline, 141,611 subjects were included. On the website, input fields were created for age, sex and all main plasma lipids. Upon input of these values, corresponding percentiles are calculated, and output is displayed in a ta-
\end{abstract}

\footnotetext{
N. S. Nurmohamed · D. Collard · E. S. G. Stroes ·

L. F. Reeskamp $(\square)$

Department of Vascular Medicine, Amsterdam University

Medical Centers, location AMC, University of Amsterdam, Amsterdam, The Netherlands

l.f.reeskamp@amsterdamumc.nl

\section{N. S. Nurmohamed}

Department of Cardiology, Amsterdam University Medical Centers, location VUMC, Vrije Universiteit Amsterdam, Amsterdam, The Netherlands
}

J. W. Balder · J. A. Kuivenhoven

Department of Paediatrics, University Medical Center Groningen, University of Groningen, Groningen, The Netherlands ble and an interactive graph for each lipid. The website has been made available in both Dutch and English and can be accessed at www.lipidtools.com. Conclusion We constructed the first searchable, national lipid reference value tool with graphical display in the Netherlands to use in screening for dyslipidaemias and to reduce the underuse of lipid-lowering therapy in Dutch primary prevention. This study illustrates that data collected in big-cohort studies can be made easily accessible with modern digital techniques and preludes the digital health revolution yet to come.

Keywords Lipids - Cholesterol - Reference values · LDL-C · CVD · Prevention

What's new?

- Lipid values are sex- and age-specific and thus require knowledge of normal value ranges for appropriate clinical care.

- We developed the first searchable, national lipid reference value tool with graphical display in the Netherlands.

- This tool can educate both physicians and patients on the normality or abnormality of lipid levels in daily cardiovascular practice and contribute towards prevention of cardiovascular disease.

- This study showed that cohort studies can be used to directly address clinical issues with the use of modern digital techniques and preludes the digital health revolution yet to come. 


\section{Introduction}

One of the most important established, causal risk factors for cardiovascular disease (CVD) is dyslipidaemia. In particular, hypercholesterolaemia-which is characterised by elevated low-density lipoprotein (LDL) cholesterol plasma levels-is causally linked to (premature) development of atherosclerosis and subsequent CVD [1]. Plasma lipid levels are routinely and frequently measured in patients with established CVD or a high CVD risk in, for example, the fields of cardiology, neurology, internal medicine and primary medicine. In Dutch primary care alone, the estimated number of yearly measured lipid profiles comprising valuable information on total cholesterol, LDL cholesterol, high-density lipoprotein (HDL) cholesterol and triglycerides currently exceeds 500,000 (data from personal communication).

The interpretation of these measured lipid values can be challenging and requires insight into reference values in the general population. Similarly to other laboratory measurements, lipid values are strongly affected by age and sex [2, 3]. Patient-specific reference values are not only needed for diagnostic decision-making (e.g. genetic testing for familial hypercholesterolaemia), but are also important for creating awareness among physicians and patients of abnormal lipid levels and overcoming the evidence-practice gap in the prescription of lipid-lowering therapies [4]. This gap between patients qualifying for lipid-lowering therapies and those receiving it, is illustrated by the fact that $77 \%$ of primary prevention patients and $31 \%$ of secondary prevention patients do not receive lipid-lowering therapy despite recommendation to do so in Dutch guidelines on cardiovascular risk management [5].

These and other practical healthcare-related difficulties may be overcome by using data that are generated in big-cohort studies. This type of cohort has been used to study the aetiology, incidence and prognosis of diseases [6], and also, for example, to determine the distribution of lipid levels among the Dutch population [2, 3]. While study outcomes are published in peer-reviewed scientific journals and implemented in guidelines, the actual improvement of clinical decision-making and patient education with this data is lagging. Only a few applications have established routine use in the consulting room, such as U-Prevent [7]. However, recent technical advances have not only enabled the generation and analysis of ever-increasing datasets [8], but also the development of easy-to-use, graphically attractive and clinically meaningful ways to use research results in actual clinical practice [9].

This report describes the development of an interactive tool based on data from a big Dutch cohort study that provided age- and sex-specific lipid reference values, in an effort to aid in diagnosis of dyslipidaemias and to address the evidence-practice gap in primary prevention.

\section{Methods}

\section{Study population}

For the current analysis, we used data from healthy adults and children in the LifeLines cohort [10], which were extracted from two studies investigating the distribution of lipid levels among the Dutch population $[2,3]$. The entire three-generational cohort consists of a representative sample comprising 167,729 persons from the northern part of the Netherlands. Individuals aged between 25 and 50 years were recruited by general practitioners, after which their partner, parents and children were also invited to participate. Adult individuals could also opt to participate through selfregistration, after which their family members were invited to participate. All participating subjects signed for informed consent.

Subjects under the age of 8 years old or with a selfreported history of CVD, defined as myocardial infarction, percutaneous coronary intervention, coronary artery bypass grafting or stroke, were excluded. In addition, participants reporting use of lipid-lowering therapies (statins, ezetimibe or fibrates) and non-fasting participants were excluded from the cohort used for the tool development.

Venous blood samples of the remaining participants were collected after an overnight fast. The laboratory procedures for measurement of total cholesterol, LDL cholesterol, HDL cholesterol and triglycerides have been described elsewhere [2].

\section{Percentile calculation}

Percentiles of lipid values of the four lipid classes were made for 5-year age groups from 20 years and older, after stratification by sex. Children $(<18$ years old) and participants aged 18 or 19 years were included as two additional age groups, stratified by sex. For each age and sex group, percentile scores were obtained by ordering the measured lipid values and subsequently assigning the relative ranking in percent rounded to the nearest half of the measured value. We repeated this procedure for every lipid category (total cholesterol, LDL cholesterol, HDL cholesterol and triglycerides).

\section{Tool development}

To interactively display these values, RStudio version 1.2.5019 and $\mathrm{R}$ version 3.6.1 were used after installation of the packages Shiny, Dplyr and Plotly (RStudio Inc., Boston, MA, USA; www.rstudio.com). Dplyr was used for data manipulation, Plotly for interactive graph development and Shiny for development of a user input-dependent and responsive interface. Next, HyperText Markup Language (HTML) and Cascading Style Sheets (CSS) were used to create a web layout. Finally, we hosted a Linux server with additional installation of the RStudio Shiny Server appli- 


\section{Advertisement placed here.}

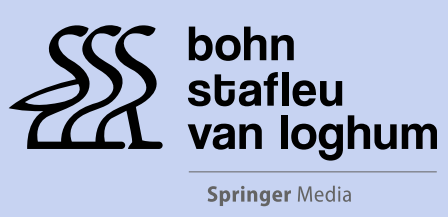

Houten 2021 


\section{Advertisement placed here.}

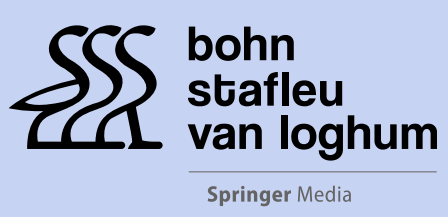

Houten 2021 


\section{Advertisement placed here.}

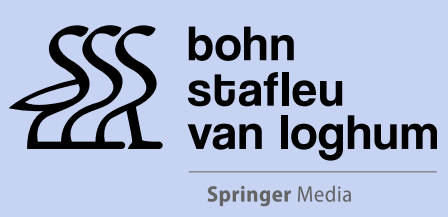

Houten 2021 


\section{Advertisement placed here.}

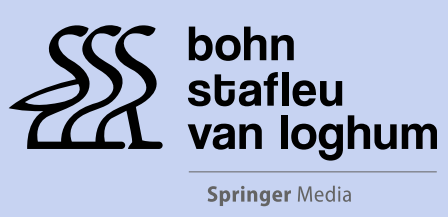

Houten 2021 


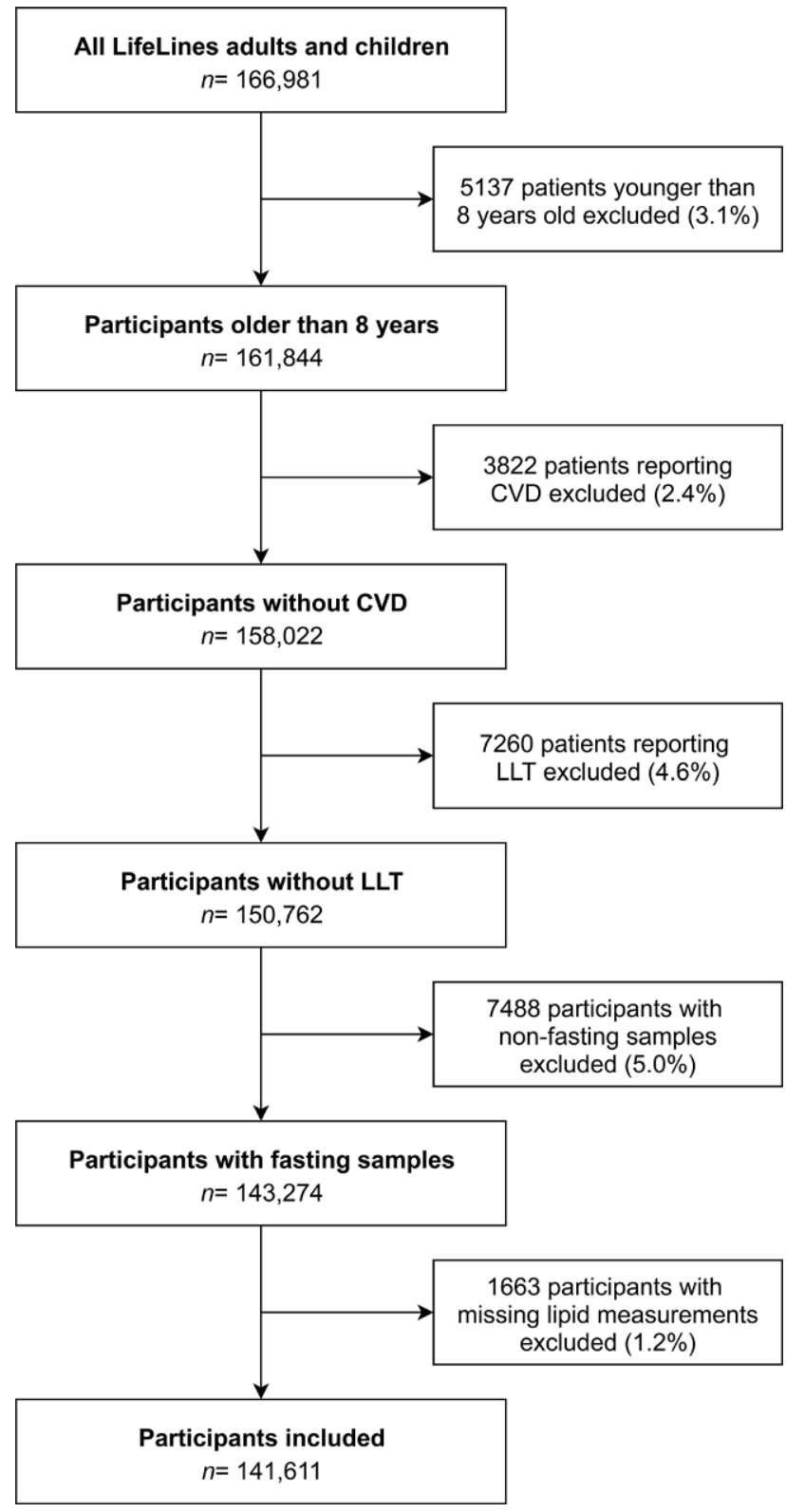

Fig. 1 Flowchart of patient inclusion for lipid percentiles. Participants younger than 8 years old, reporting cardiovascular disease (CVD), reporting use of lipid-lowering therapy (LLT), with non-fasting samples or with missing lipid measurements were excluded

cation. A web server was configured to make the application freely available to the public.

\section{Results}

Of the 166,981 adults and children in the LifeLines cohort, 5137 participants $(3.1 \%)$ younger than 8 years old were excluded (Fig. 1). In addition, the following participants were excluded: 3822 (2.4\%) who had a CVD history, 7260 (4.6\%) who were on lipid-lowering therapy, $7488(5.0 \%)$ due to non-fasting samples and $1663(1.2 \%)$ due to missing lipid measurements. After exclusion of a total of 25,370 participants, 141,611 par-
Table 1 Number of included participants per age group, divided by sex

\begin{tabular}{|l|r|r|r|}
\hline Age, years & Males & Females & \multicolumn{1}{|c|}{ Total } \\
\hline$<18$ & 3823 & 4248 & 8071 \\
\hline $18-19$ & 842 & 1616 & 2458 \\
\hline $20-24$ & 1905 & 3995 & 5900 \\
\hline $25-29$ & 4638 & 6576 & 11,214 \\
\hline $30-34$ & 5302 & 7059 & 12,361 \\
\hline $35-39$ & 6485 & 9535 & 16,020 \\
\hline $40-44$ & 8822 & 13,129 & 21,951 \\
\hline $45-49$ & 10,049 & 14,789 & 24,838 \\
\hline $50-54$ & 5705 & 8289 & 13,994 \\
\hline $55-59$ & 3338 & 5166 & 8504 \\
\hline $60-64$ & 3227 & 4502 & 7729 \\
\hline $65-69$ & 2182 & 2794 & 4976 \\
\hline $70-74$ & 1013 & 1284 & 2297 \\
\hline $75-79$ & 383 & 520 & 903 \\
\hline$\geq 80$ & 174 & 221 & 395 \\
\hline Total & 57,888 & 83,723 & 141,611 \\
\hline
\end{tabular}

ticipants (133,540 adults and 8071 children) were included in the current study. Numbers of included participants per age group are presented in Tab. 1. The 5th and 95th percentiles for total cholesterol, LDL cholesterol, HDL cholesterol and triglycerides per age and sex group are shown in Fig. 2.

Following the calculation of percentiles per group, we created input fields for total cholesterol, LDL cholesterol, HDL cholesterol, triglycerides, age, sex and unit of cholesterol measurements (i.e. mmol/L or $\mathrm{mg} / \mathrm{dL}$ ). After a user has filled in these fields, the output is formatted in a table displaying the plasma concentrations and corresponding percentiles. Simultaneously, output is displayed in an interactive graph for each plasma lipid feature separately. We ensured that the output instantly adapts to changes in the input boxes.

Using an HTML template with CSS styling, we created a clear and simple responsive layout suitable for mobile and web use. After installing Shiny Server, we made the application available in both Dutch and English at www.lipidtools.com (Fig. 3), via the Dutch Foundation for Familial Hypercholesterolemia cascade screening programme (Stichting Landelijk Expertisecentrum Erfelijkheidsonderzoek Familiaire Hart- en Vaatziekten; www.leefh.nl) and at www.jojogenetics.nl.

\section{Discussion}

We provided the first searchable, national lipid reference values in the Netherlands, which can be used in clinical cardiovascular practice, particularly in primary prevention. Our study showed that data collected in large-cohort studies can be made easily accessible owing to rapid advances in digital tools 


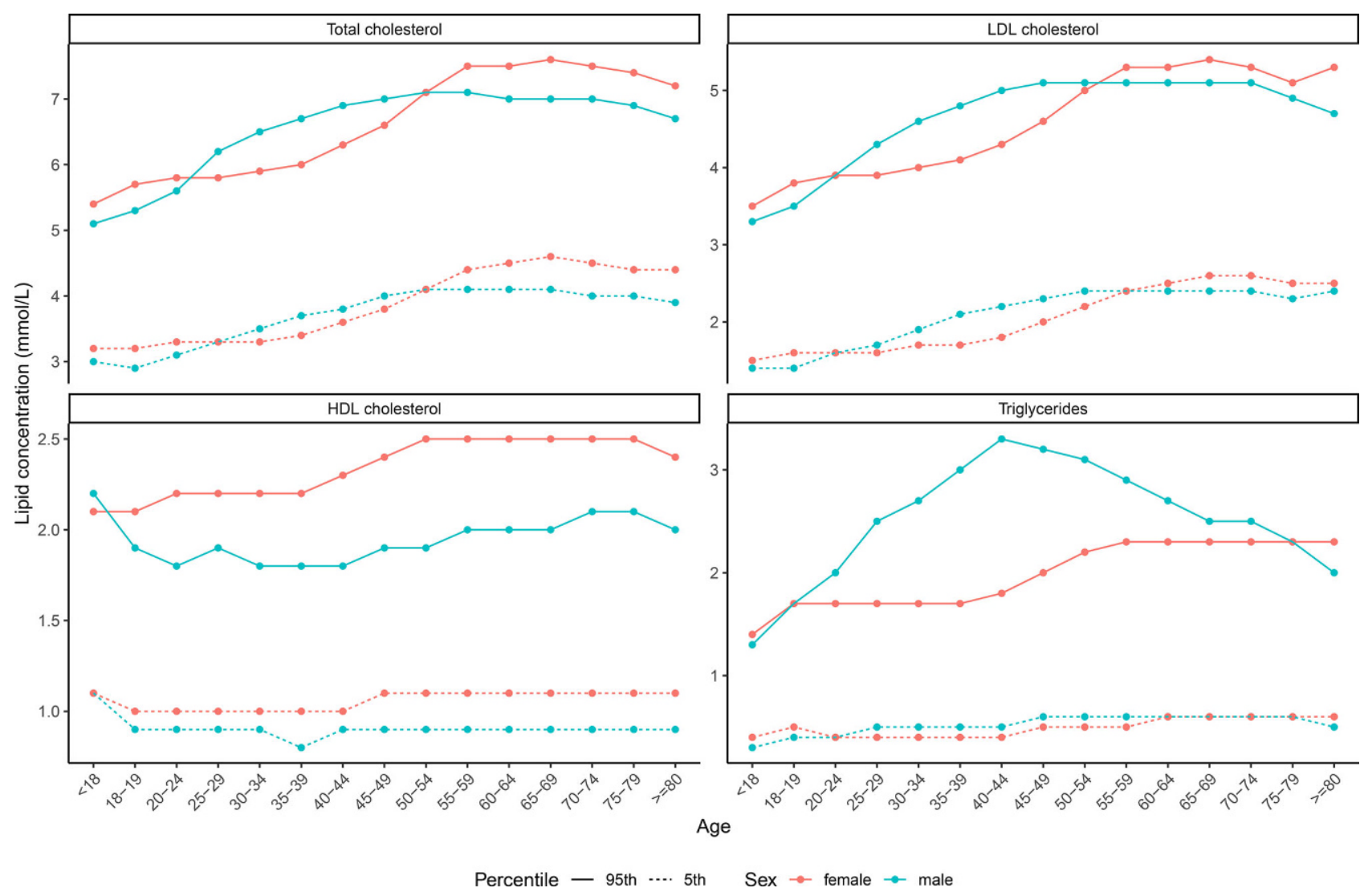

Fig. 2 Fifth and ninety-fifth percentiles for total cholesterol, LDL cholesterol, HDL cholesterol and triglycerides (in $\mathrm{mmol} / \mathrm{L}$ ) per sex (male/female) and per age group (x-axis)

that combine simple statistical methods with standard programming languages.

Up to recently, non-searchable, outdated cohort data collected in the 1980s in the USA were used to provide lipid percentiles to Dutch physicians [11]. However, demographics of the current Dutch population, such as lifestyle, ethnicity and genetic factors, are largely different than those of the American population four decades ago, leading to a demand for upto-date nation-specific lipid percentiles.

The availability of reference lipid percentile values is particularly important for diagnosing dyslipidaemias, since lipid values in all lipid traits change over the course of a lifetime with different magnitudes for both sexes, making it nearly impossible to know them by heart or to capture them in one table $[2,3]$. The best-known genetic dyslipidaemia, for which specific percentiles play a pivotal role in diagnosis, is familial hypercholesterolaemia. Knowing whether a patient's LDL cholesterol is high for his or her respective age and sex can raise the suspicion of this disease. The 95th percentile for the age- and sexcorrected LDL cholesterol value of family members is also required when the Dutch Lipid Clinic Network criteria are used to make the diagnosis of familial hypercholesterolaemia $[12,13]$. The use of up-to-date age- and sex-specific percentiles will support a more accurate diagnosis.
In addition to providing more accurate and up-todate reference values and thereby helping in the diagnosis of dyslipidaemias, our tool can contribute to increasing physicians' awareness of lipid abnormalities. Ultimately, this could contribute to reducing the evidence-practice gap in lipid-lowering therapy use in the Netherlands $[4,5]$.

Epidemiological cohort studies have been performed for decades and are nowadays containing ever-increasing numbers of included patients; for example, the UK Biobank comprises data from $>500,000$ participants [14]. Although results from these studies often form the basis for a better understanding of health and disease, most underlying data and statistical models are not accessible for use in clinical practice. For example, patterns in blood markers (such as lipids) or regression models predicting disease are widely published, but they are not easily incorporated in clinical care.

In general, our study showed that data collected in large-cohort studies can be made easily accessible with a combination of current digital tools. For example, these models could be incorporated in electronic health records [15] or, with the addition of easyto-understand information and explanation, be made available for use by patients themselves [7]. We foresee that continuous developments in the digital health 
Fig. 3 Screenshot from lipid reference tool in which obtained lipid percentiles are made publicly available. The tool consists of multiple input fields (i.e. lipid levels, age and sex) and multiple output formats. In the top right table, the lipid value and corresponding age- and sex-specific percentile are displayed. In the bottom half, a lipid traitspecific percentile graph is shown with a marker indicating the percentile based on the entered lipid values. The user can select a different lipid percentile graph using the tabs on top of the graph

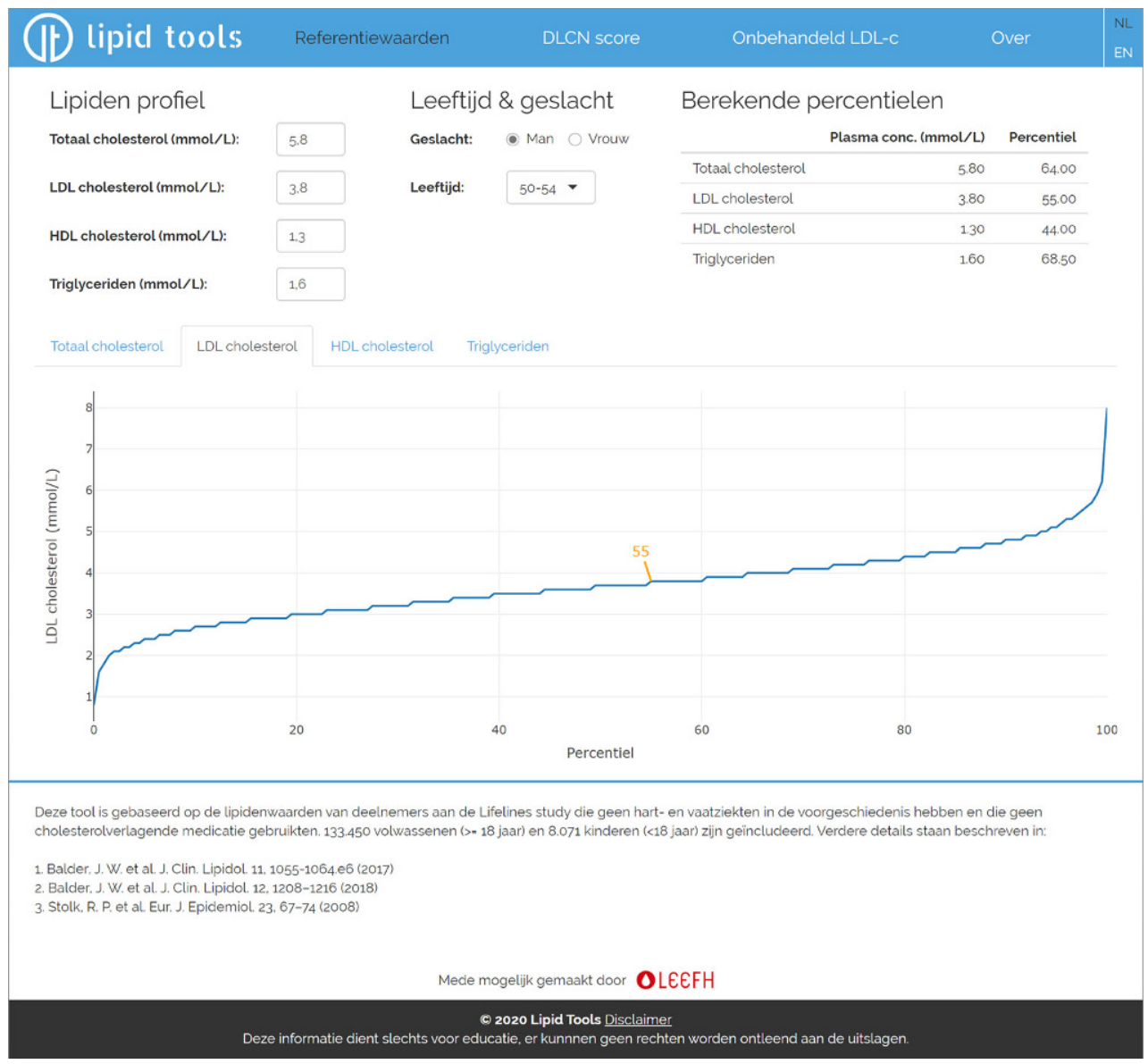

arena will lead to an accelerated implementation of such research outcomes in clinical care.

\section{Limitations}

Our study has several limitations. First, it has been established that there are ethnic differences in lipid levels in the Netherlands [16]. As the LifeLines cohort consists of habitants from the three Northern Dutch provinces, more than $98 \%$ of the study population is of Caucasian/ West-European descent [17], making it uncertain whether these reference values can be applied to other ethnic groups.

Second, the cohort did not include secondary prevention patients. However, the proportion of patients excluded based on their CVD history was small (2.4\%), which could have resulted in only a very small effect on the obtained percentiles, especially in younger individuals. Furthermore, this exclusion does not affect use of the tool in primary prevention, and comparison with healthy individuals could also be of use in secondary prevention.

Last, self-reported data were used for the history of CVD or lipid-lowering drug use, which resulted in a small risk of data imprecision.

\section{Conclusion}

We developed the first clinically useful web-based tool that provides easy searchable and interactively displayed Dutch lipid profile percentiles, which can educate physicians on the normality or abnormality of lipid levels in clinical practice, particularly in primary prevention. This is not only of particular interest for dyslipidaemia screening in the Netherlands, but we also hope to provide a small step towards reducing the evidence-practice gap, mainly in primary prevention. In general, this study illustrates that data collected in big-cohort studies can be made easily accessible with modern digital techniques and preludes the digital health revolution yet to come.

Acknowledgements We would like to thank all participants of the LifeLines cohort for participating in this study, as well as the LifeLines biobank for allowing us to use its cohort data for the development of these tools.

Conflict of interest N.S. Nurmohamed and L.F. Reeskamp are cofounders of Lipid Tools and have received funding for the development and maintenance of the lipid percentile tools from the Dutch Foundation for Familial Hypercholesterolemia cascade screening (Stichting Landelijk Expertisecentrum Erfelijkheidsonderzoek Familiaire Hart- en Vaatziekten; LEEFH). E.S.G. Stroes is medical director at Stichting LEEFH and reports advisory board/lecturing fees paid to the institution 
from Amgen, Sanofi-Regeneron, Mylan, Esperion and Novartis, and grants from Athera, all outside the submitted work. D. Collard, J.W. Balder and J.A. Kuivenhoven declare that they have no competing interests.

Open Access This article is licensed under a Creative Commons Attribution 4.0 International License, which permits use, sharing, adaptation, distribution and reproduction in any medium or format, as long as you give appropriate credit to the original author(s) and the source, provide a link to the Creative Commons licence, and indicate if changes were made. The images or other third party material in this article are included in the article's Creative Commons licence, unless indicated otherwise in a credit line to the material. If material is not included in the article's Creative Commons licence and your intended use is not permitted by statutory regulation or exceeds the permitted use, you will need to obtain permission directly from the copyright holder. To view a copy of this licence, visit http://creativecommons.org/licenses/by/4.0/.

\section{References}

1. Ference BA, Ginsberg HN, Graham I, et al. Low-density lipoproteins cause atherosclerotic cardiovascular disease. 1. Evidence from genetic, epidemiologic, and clinical studies. A consensus statement fromthe European Atherosclerosis Society Consensus Panel. Eur Heart J. 2017;38:2459-72.

2. Balder JW, de Vries JK, Nolte IM, Lansberg PJ, Kuivenhoven JA, Kamphuisen PW. Lipid and lipoprotein reference values from 133,450 Dutch Lifelines participants: age- and gender-specific baseline lipid values and percentiles. JClin Lipidol. 2017;11:1055-1064.e6.

3. Balder JW, Lansberg PJ, Hof MH, Wiegman A, Hutten BA, Kuivenhoven JA. Pediatric lipid reference values in the general population: the Dutch lifelines cohort study. JClin Lipidol. 2018;12:1208-16.

4. Nieuwlaat R, Schwalm JD, Khatib R, Yusuf S. Why are we failing to implement effective therapies in cardiovascular disease? Eur Heart J. 2013;34:1262-9.

5. Balder JW, Scholtens S, de Vries JK, et al. Adherence to guidelines to prevent cardiovascular diseases: the lifelines cohort study. Neth J Med. 2015;73:316-23.

6. Byles J. Making innovative use of cohort data. Public Health Res Pract. 2016; https://doi.org/10.17061/phrp2631627.
7. Jaspers NEM, Blaha MJ, Matsushita K, et al. Prediction of individualized lifetime benefit from cholesterol lowering, blood pressure lowering, antithrombotic therapy, and smoking cessation in apparently healthy people. Eur Heart J.2020;41:1190-9.

8. Alaa AM, Bolton T, Di Angelantonio E, Rudd JHF, van der Schaar M. Cardiovascular disease risk prediction using automated machine learning: a prospective study of 423,604 UKBiobank participants. Plos One. 2019;14:e213653.

9. Doi J, Potter G, Wong J, et al. Web application teaching tools for statistics using $\mathrm{R}$ and shiny. Technol Innov Stat Educ. 2016;9:32.

10. Scholtens S, Smidt N, Swertz MA, et al. Cohort Profile: LifeLines, a three-generation cohort study and biobank. Int JEpidemiol. 2015;44:1172-80.

11. Gotto AM, Bierman EL, Connor WE, et al. Recommendations for treatment of hyperlipidemia in adults. A joint statement of the nutritism committee and the council on arteriosclerosis. Circulation. 1984;69:1065A-90A.

12. Nordestgaard BG, Chapman MJ, Humphries SE, et al. Familial hypercholesterolaemia is underdiagnosed and undertreated in the general population: guidance for clinicians to prevent coronary heart disease. Eur Heart J. 2013;34:3478-90a.

13. Nederlandse Internisten Vereniging. Richtlijn Erfelijke Dyslipidemie in de 2e en 3e lijn. 2018. https:// richtlijnendatabase.nl/richtlijn/erfelijke_dyslipidemie in_de_2e_en_3e_lijn/startpagina_dyslipidemie.html. Accessed 2 Apr 2020.

14. Sudlow C, Gallacher J, Allen N, et al. UK Biobank: an open access resource for identifying the causes of a wide range of complex diseases of middle and old age. PLoS Med. 2015;12:e1001779.

15. Myers KD, Knowles JW, Staszak D, et al. Precision screening for familial hypercholesterolaemia: a machine learning study applied to electronic health encounter data. Lancet. 2019;1:e393-e402.

16. Gazzola K, Snijder MB, Hovingh GK, Stroes ESG, Peters RJG, van den Born BJH. Ethnic differences in plasma lipid levels in a large multiethnic cohort: The HELIUS study. J Clin Lipidol. 2018;12:1217-1224.e1.

17. Klijs B, Scholtens S, Mandemakers JJ, Snieder H, Stolk RP, Smidt N. Representativeness of the LifeLines cohort study. Plos One. 2015;10:e137203. 